\title{
Evaluation of cortical bone thickness of mandible with cone beam computed tomography for orthodontic mini implant installation
}

Seyed Hossein Moslemzade ${ }^{1}$, Yusef Kananizadeh ${ }^{2}$, Amin Nourizadeh $^{3 *}$, Aydin Sohrabi $^{1}$, Mehrdad Panjnoosh ${ }^{4}$, Elnaz Shafiee ${ }^{5}$

1. Department of Orthodontics, Faculty of Dentistry, Tabriz University of Medical Sciences, Tabriz, Iran

2. Department of Oral and maxillofacial Surgery, Faculty of Dentistry, Tabriz University of Medical Sciences, Tabriz, Iran

3. Department of Prosthodontics, Faculty of Dentistry, Tabriz University of Medical Sciences, Tabriz, Iran

4. Department of Oral and Maxillofacial Radiology, Faculty of Dentistry, Tehran University of Medical Sciences, Tehran, Iran

5. Department of Prosthodontics, Faculty of Dentistry, Islamic Azad University of Tehran, Tehran, Iran

\begin{abstract}
Background: Achieving maximum anchorage without movement of the teeth in anchorage unit has been a great challenge in orthodontics and the success of the treatment plan highly depends on it. In this case, using orthodontic mini-implants can make a huge difference. The objective of this retrospective study was to measure thickness of cortical bone at prospective mini-implant placement sites in mandible in order to understand stability aspects of miniimplant placement by using cone-beam computed tomography (CBCT) images.

Materials and Methods: Initial 3-dimensional images of 40 adult patients were studied. The cortical bone thickness was obtained at the alveolar processes from canine to second molar at 5 different vertical levels from the cementoenamel junction (CEJ). To determine the cortical bone thickness, tangent lines were drawn buccolingually to the roots in axial section and a third line was drawn from the middle of these two lines, and the cortical bone thickness was measured where the third line crossed the buccal cortex.

Results: Mandibular and buccal cortical bone thicknesses were 0.79 to $2.49 \mathrm{~mm}$, respectively. There was a statistically significant increase from the CEJ to the apex $(P<0.001)$, while this increase was not statically significant at interdental area of teeth \#3 and \#4. Comparing the 4 $\mathrm{mm}$ section in all sites showed significant increase from anterior to posterior.

Conclusion: Based on our results, the cortical bone thickness mostly follows a pattern and depends on the interradicular site rather than individual differences.
\end{abstract}

Key words: Cone-beam computed tomography; orthodontic anchorage procedures

\section{Corresponding author:}

Amin Nourizadeh

Department of Prosthodontics, Faculty of Dentistry, Tabriz University of Medical Sciences, Tabriz, Iran Email: nourizadeh.amin@gmail.com Tel: +989144129518

Receive date: 2014-05-25 | Accept date: 2014-06-25 | Publish date:

2014-07-04 DOI: 10.7575/aiac.abcmed.14.02.02.10 


\section{Introduction}

Use of an appropriate anchorage is one of the important factors in achieving successful results in fixed orthodontics. Conventionally, inclusion of more teeth in the anchorage unit or use of extra oral appliances to strengthen the anchorage unit is common (1). Success in extra oral anchorage techniques depends on patient cooperation and with the use of these techniques, even under the best condition, some unfavorable movements usually occur in the anchorage unit (2). Therefore, provision of an appropriate anchorage has been one of the main concerns in orthodontic treatment planning until 1997, when Kanomi reported the use of orthodontic mini-implants for tooth movements, providing an all-bone anchorage and easy placement and removal (3). It is possible to move the teeth accurately and without limitations with the use of such miniimplants (1). One of the challenges in relation to the use of mini-implants is to determine an appropriate and exact location for these miniimplants. Various criteria have been defined in this context including Nanda's works, consisting of compatibility, biomechanical design, sufficient attention not to inflict traumas to roots, arteries, veins and nerves, and lastly adequate thickness of the cortical bone for the stability of the implant (4).

Different studies have been carried out to standardize these criteria with the use of different techniques to determine an appropriate location to place mini-implants. Such efforts have mainly focused on the shape, and form or primary stability. Some of these studies have emphasized measurements and evaluation of anatomic locations for proper placement of these mini-implants (1) In recent years, cone-beam computed tomography (CBCT) technique has been used extensively in orthodontics, implant dentistry, diagnosis of head and neck lesions and determining the location of mini-implants (5-7). CBCT can provide thinner cross-sections compared to spiral CT (3). A large number of studies have shown that the cortical bone thickness is a vital factor in achieving stability for mini-implants (812). However, there are only limited studies in relation to measurement of the cortical bone thickness and comparison of various areas and cross-sections and the results are contradictory (13-15).

Since there is no comprehensive study available on the cortical bone thickness in different interdental areas and at different cross-sections, the present study was designed. In the present study the CBCT image archives were used retrospectively to determine the cortical bone thickness at interdental areas between canines, first premolars, second premolars, first molars and second molars in the mandible.

\section{Materials and Methods}

The present restorative study was carried out using the CBCT image archives of a private oral and maxillofacial radiology center in Tehran, Iran. The subjects consisted of patients referred to the center for $\mathrm{CBCT}$ examinations. Using the Altman normogram at a power of $80 \%, \alpha=0.05$ and a standard difference of 0.9 , the sample size was calculated at 38 ; however 40 samples were included to increase the power of the study. Sampling was carried out by random participation method. Inclusion criteria included presence of all the teeth in the mandible (presence of third molars was not necessary), no orthodontic treatment before CBCT examination, and absence of any rotation and developmental malformations of the teeth. Exclusion criteria were presence of horizontal alveolar bone loss measuring more than $2 \mathrm{~mm}$ in relation to the CEJ, presence of crowding measuring more than $2 \mathrm{~mm}$, presence of spacing in the mandibular arch, and women over 40 years of age. 
Planmeca ProMax 3D CBCT unit (Finland) was used to take CBCT images at $k V p=84$, effective exposure $=6$ seconds and $m A=2$. The three-dimensional images were evaluated by Planmeca Romexis Viewer 2.0.1 software in 5 axial cross-sections at 2-, 4-, 6-, 8- and 10-mm distances from the CEJ under the supervision of an oromaxillofacial radiologist (Figures 1 and 2). First, lines were drawn tangential to the roots (Figure 3) resulting in two situations for measuring the cortical bone thickness: (a) the lines drawn tangential to the roots were almost parallel; in this situation a line parallel to the tangential lines was drawn from the middle of the minimum distance between the roots and the thickness was measured and recorded at the intersection of the line drawn parallel to the cortex, and (b) The lines drawn tangential to the roots were not parallel and an angle was formed between them. In this situation, the thickness was measured and recorded at the intersection of the bisector of the angle with the cortex.

Data were analyzed using descriptive statistical methods (means \pm standard deviation) separately for each interdental area at different cross-sections. KolmogorovSmirnov test was used to evaluate normal distribution of data. Friedman's test was used for analysis of data not distributed normally. In cases in which the differences were significant, post hoc Wilcoxon signed ranks test was used. All the analyses were carried out using SPSS 17. Statistical significance was set at $P<0.05$.

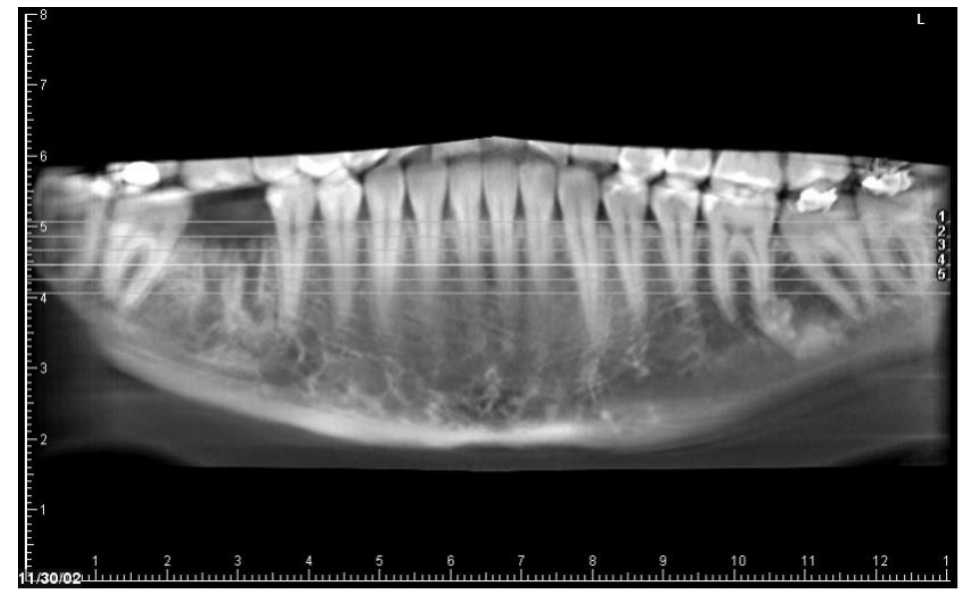

Figure 1: The panoramic view of the mandible 


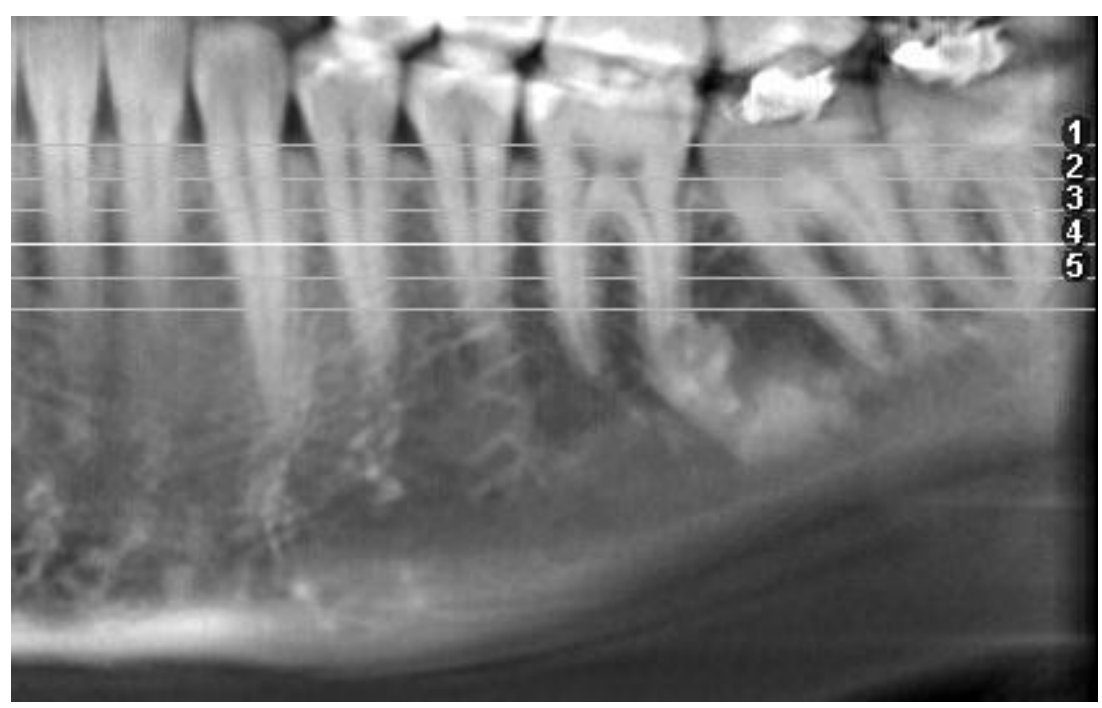

Figure 2: 2-mm coronal cross-sections from the CEJ

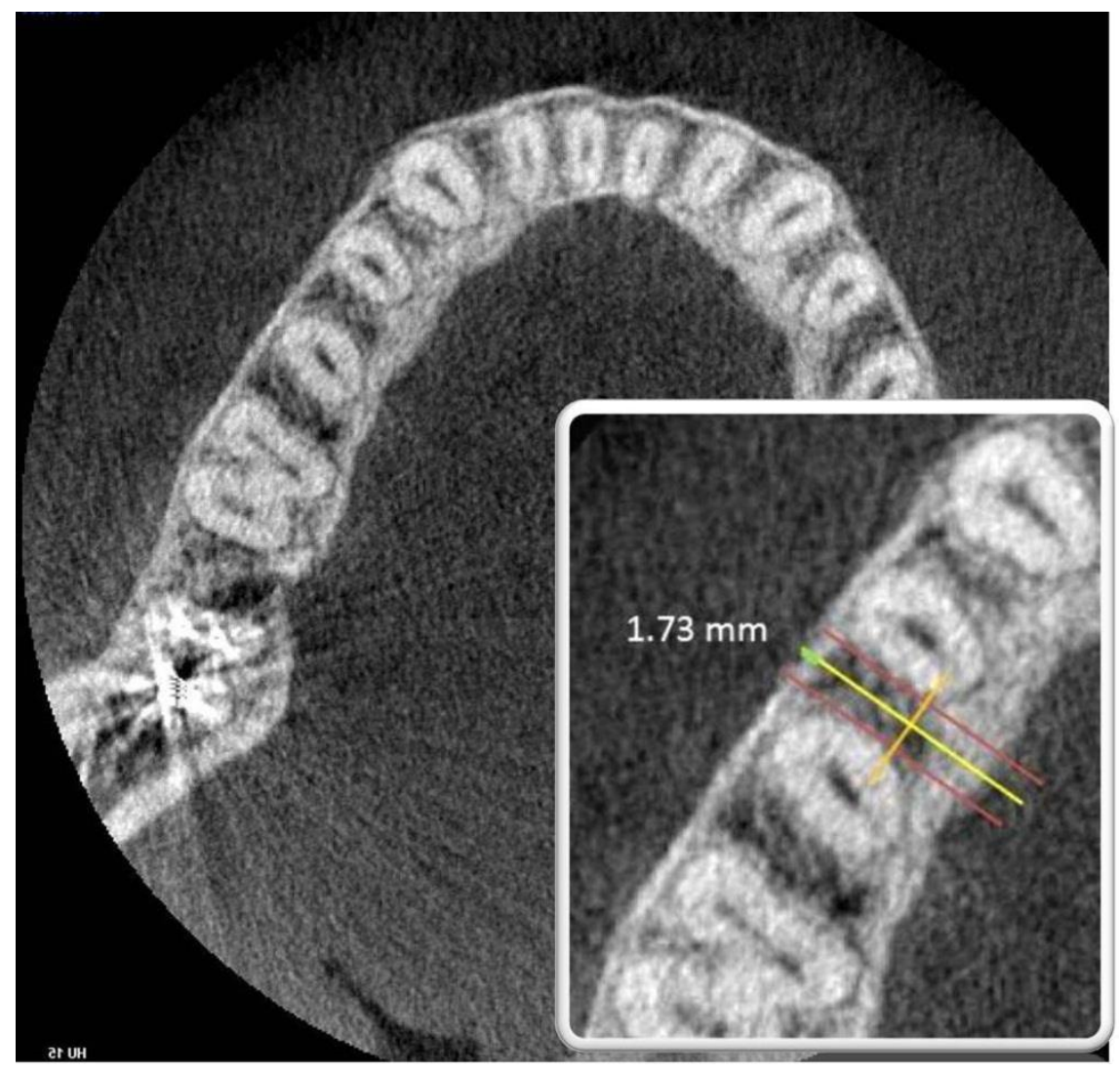

Figure 3: An axial view at a distance of $4 \mathrm{~mm}$ from the CEJ 


\section{Results}

Of 40 subjects included in the present study, 11 (27.5\%) were male and 29 (72.5\%) were female. Table 1 presents the descriptive data in relation to the measurement of cortical bone thickness at 5 cross-sections at 2-, 4-, 6-, 8- and 10-mm distances from the CEJ at 4 different interdental areas between the teeth \#3 and \#4,
$\# 4$ and \#5, \#5 and \#6, and \#6 and \#7.

Comparison of cortical bone thickness at different interdental areas between the teeth \#3 and \#4 showed significant differences between 4- and 6-mm sections and between 8 and $10-$

\begin{tabular}{lccccccc}
\hline \multicolumn{1}{c}{ Location* } & Variable data & Lost data & Median & Mean & SD & Min & Max \\
\hline C.34.2 mm & 21 & 19 & 0.700 & 0.792 & 0.4300 & 0.3 & 1.9 \\
C.34.4 mm & 40 & 0 & 0.750 & 0.968 & 0.3729 & 0.2 & 1.7 \\
C.34.6 mm & 40 & 0 & 0.950 & 1.217 & 0.3769 & 0.3 & 1.7 \\
C.34.8 mm & 40 & 0 & 1.000 & 1.292 & 0.3711 & 0.4 & 1.9 \\
C.34.10 mm & 40 & 0 & 1.400 & 1.517 & 0.3768 & 0.4 & 2.3 \\
C. $45.2 \mathrm{~mm}$ & 27 & 13 & 0.900 & 1.067 & 0.4509 & 0.2 & 1.6 \\
C. $45.4 \mathrm{~mm}$ & 40 & 0 & 1.100 & 1.283 & 0.4255 & 0.2 & 1.8 \\
C. $45.6 \mathrm{~mm}$ & 40 & 0 & 1.200 & 1.433 & 0.4214 & 0.6 & 2.2 \\
C. $45.8 \mathrm{~mm}$ & 40 & 0 & 1.400 & 1.533 & 0.4329 & 0.7 & 2.3 \\
C. $45.10 \mathrm{~mm}$ & 40 & 0 & 1.400 & 1.650 & 0.4695 & 0.7 & 2.6 \\
C.56.2 mm & 31 & 9 & 1.100 & 1.233 & 0.4320 & 0.3 & 2.1 \\
C.56.4mm & 40 & 0 & 1.200 & 1.392 & 0.3882 & 0.5 & 1.9 \\
C.56.6 mm & 40 & 0 & 1.450 & 1.525 & 0.4901 & 0.7 & 2.5 \\
C.56.8 mm & 40 & 0 & 1.600 & 1.758 & 0.5041 & 0.6 & 3.1 \\
C.56.10 mm & 40 & 0 & 1.900 & 2.067 & 0.6164 & 1.1 & 3.8 \\
C.67.2 mm & 35 & 5 & 1.300 & 2.525 & 0.7158 & 0.3 & 3.8 \\
C.67.4 mm & 40 & 0 & 2.100 & 2.108 & 0.7365 & 0.3 & 4.0 \\
C. $67.6 \mathrm{~mm}$ & 40 & 0 & 2.200 & 2.392 & 0.7583 & 0.9 & 4.5 \\
C.67.8 mm & 40 & 0 & 2.300 & 2.458 & 0.7635 & 1.1 & 4.4 \\
C.67.10 mm & 40 & 0 & 2.500 & 2.492 & 0.6304 & 1.2 & 4.1 \\
\hline
\end{tabular}

Table 1: The results of descriptive statistics

*Measurements have been shown with abbreviations; e.g., C.34.2 mm (C, cortical bone thickness; 34, between teeth $\# 3$ and \#4 (the canine and first premolar); $2 \mathrm{~mm}$, at a distance of $2 \mathrm{~mm}$ from the CEJ. 
$\mathrm{mm}$ sections $(\mathrm{P}<0.001)$. Evaluation of cortical bone thickness at interdental areas of the teeth $\# 4$ and \#5, and \#5 and \#6 showed that cortical bone thickness at each area and between all the cross-sections which were compared two- by-two in each area demonstrated statistically significant differences, and the thickness increased linearly with an increase in distance from the CEJ $(P<0.001)$.

\begin{tabular}{|c|c|c|c|c|c|}
\hline Location & Variable & Lost data & Mean & $90 \%$ of subjects & $80 \%$ of subjects \\
\hline C. $34.2 \mathrm{~mm}$ & 21 & 19 & 0.792 & 0.558 & 0.614 \\
\hline C. $34.4 \mathrm{~mm}$ & 40 & 0 & 0.968 & 0.753 & 0.802 \\
\hline C.34.6 mm & 40 & 0 & 1.217 & 1.056 & 1.065 \\
\hline C. $34.8 \mathrm{~mm}$ & 40 & 0 & 1.292 & 1.120 & 1.161 \\
\hline C. $34.10 \mathrm{~mm}$ & 40 & 0 & 1.517 & 1.347 & 1.388 \\
\hline C. $45.2 \mathrm{~mm}$ & 27 & 13 & 1.067 & 0.854 & 0.905 \\
\hline C. $45.4 \mathrm{~mm}$ & 40 & 0 & 1.283 & 1.129 & 1.166 \\
\hline C. $45.6 \mathrm{~mm}$ & 40 & 0 & 1.433 & 1.200 & 1.256 \\
\hline C. $45.8 \mathrm{~mm}$ & 40 & 0 & 1.533 & 1.296 & 1.353 \\
\hline C. $45.10 \mathrm{~mm}$ & 40 & 0 & 1.650 & 1.401 & 1.461 \\
\hline C. $56.2 \mathrm{~mm}$ & 31 & 9 & 1.233 & 1.030 & 1.079 \\
\hline C.56.4 mm & 40 & 0 & 1.392 & 1.243 & 1.279 \\
\hline C. $56.6 \mathrm{~mm}$ & 40 & 0 & 1.525 & 1.240 & 1.309 \\
\hline C. $56.8 \mathrm{~mm}$ & 40 & 0 & 1.758 & 1.546 & 1.597 \\
\hline C. $56.10 \mathrm{~mm}$ & 40 & 0 & 2.067 & 1.734 & 1.814 \\
\hline C. $67.2 \mathrm{~mm}$ & 35 & 5 & 2.525 & 1.084 & 1.191 \\
\hline C. $67.4 \mathrm{~mm}$ & 40 & 0 & 2.108 & 1.711 & 1.807 \\
\hline C. $67.6 \mathrm{~mm}$ & 40 & 0 & 2.392 & 1.947 & 2.054 \\
\hline C. $67.8 \mathrm{~mm}$ & 40 & 0 & 2.458 & 2.006 & 2.115 \\
\hline C. $67.10 \mathrm{~mm}$ & 40 & 0 & 2.492 & 2.138 & 2.223 \\
\hline
\end{tabular}

Table 2: Estimation of the thickness of cortical bone at different areas in different cross-sections

Comparison of cortical bone thickness at different cross-sections between teeth \#6 and \#7 showed significant differences in cortical bone thickness between 2- and 4- $\mathrm{mm}$ distances, 6- and 8- $\mathrm{mm}$ distances and 8- and $10-\mathrm{mm}$ distances from the CEJ; however, the difference was not significant at 4- and 6-mm distance $(\mathrm{P}<0.001)$. Table 2 was prepared in order to make better clinical use of the results and to estimate cortical bone thickness in different areas and in different cross-sections at $80 \%$ and $90 \%$ confidence rates. For example, at a distance of $8 \mathrm{~mm}$ from the CEJ between canines and first premolars, in $90 \%$ and $80 \%$ of

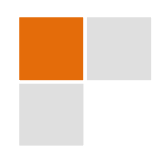


the subjects the cortical bone thicknesses were 1.120 and $1.161 \mathrm{~mm}$, respectively.

All the subjects had cortical bone at a distance of $4 \mathrm{~mm}$ from the CEJ. This crosssection is usually in the area of keratinized gingiva which is necessary for the placement of mini-implants. Therefore, the cortical bone thickness was compared in different areas and at $4-\mathrm{mm}$ cross-sections. The results showed significant differences between these areas; two-by-two comparison of the areas showed that the cortical bone thickness increased from the anterior area to the posterior area $(P<0.001)$.

\section{Discussion}

Provision of an appropriate anchorage has always been the focus of attention of orthodontists; however, the conventional methods of achieving anchorage have been associated with problems (16) In areas in which active forces are applied, the reactive forces result in tooth movement in the opposite direction. One of the techniques to decrease unfavorable tooth movements is to include more teeth in the anchorage unit, although such a technique is associated with some limitations (17). Of course, it is sometimes possible to use extraoral appliances to increase anchorage, which is not always successful as it depends on patient's compliance. Kuhlberg and Burston proposed a technique to solve the problem of anchorage $(18,19)$. Considering the fact that tipping movement is easier than bodily movement or root movement, they replaced bodily movement in the anchorage unit by tipping movement in the movement unit. Nevertheless, this technique is not applicable in semi-edentulous patients or in patients in whom the teeth should only move in one direction.

Based on the problems discussed above, anchorages independent from teeth, but within the oral cavity, were introduced including miniimplants (20). Since these mini-implants traverse through the soft and hard tissues, the thicknesses of soft tissues and cortical bone have a vital role in their success. In a study conducted by Cho and Park in 2009, (15) the thickness of mandibular cortical bone was 1.25$2.98 \mathrm{~mm}$ at a distance of 5-9 $\mathrm{mm}$ from the CEJ, increasing from the CEJ toward the apex. In the present study, the thickness was 0.781-2.647 $\mathrm{mm}$. The difference between the two studies might be attributed to the measurements made at different distances from the CEJ; measurements in the present study were made at distances of 2-15 $\mathrm{mm}$ from the CEJ.

Lim et al. (14) evaluated cortical bone thickness and distances between the roots at interdental areas in both jaws. They reported that cortical bone thickness increased from the anterior areas to the posterior areas and the thickest cortical bone was located at a distance of $4 \mathrm{~mm}$ from the gingival crest. This finding might be attributed to the morphology of ridge crest as it is convex in shape and the thickness measured is higher than the range reported in the present study and other previous studies. In a study by Baumgaertel et al. (13) to evaluate the cortical bone thickness in the buccal area for the placement of mini-implants, 30 dry skulls were evaluated by the CBCT technique. The results showed a higher cortical bone thickness in posterior areas and the thickness increased by moving away from the bone crest, consistent with the results of the present study.

Deguchi and colleagues carried out a study for qualitative evaluation of the cortical bone thickness by CBCT technique to place orthodontic implants (8). The cortical bone thickness was measured on 3-dimensional CBCT images of patients on the buccal and lingual sides on different areas at two cross-sections. The results showed that the cortical bone thickness was $1.8-2 \mathrm{~mm}$ at the apical and occlusal areas between teeth \#5 and \#6, and

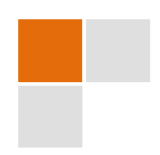


between teeth \#6 and \#7, with no significant differences between different areas of the mandible (8). In the present study, the cortical bone thickness was in a similar range at 4- and 8-mm cross-sections at \#5-6 and \#6-7 interdental areas, which is almost similar to those evaluated by Deguchi et al. (8) In addition, in the study by Deguchi and coworkers, significant differences were reported between different areas of the mandible, which might be attributed to the limited evaluations carried out in relation to both the number of subjects and the crosssections. In the present study, the cortical bone thickness was evaluated in a larger sample size and in 5 cross-sections and significant differences were observed between different areas of the mandible. In addition, in the present study, the cortical bone thickness was estimated with different confidence coefficients for each area in the subjects; no such data have been reported in the previous studies.

\section{Conclusion}

The results of the present study showed that cortical bone thickness in the mandible increased from the distal aspect of the canine to the mesial aspect of the second molar, except for the interdental area of teeth \#3 and $\# 4$, from the cervical area to the apical area. At the 4-mm cross-section, cortical bone thickness at different areas of the mandible exhibited significant differences and increased from the anterior area to the posterior area.

\section{References}

1. Lee JS, Kim JK, Park Y-C, Vanarsdall RL. Applications of orthodontic mini implants. Quintessence Publishing Company; 2007.

2. Sugawara J. A bioefficient skeletal anchorage system. Biomechanics and Esthetic Strategies in Clinical Orthodontics 2005:295-309.

3. Kim SH, Choi YS, Hwang EH, Chung KR, Kook YA, Nelson G. Surgical positioning of orthodontic mini-implants with guides fabricated on models replicated with cone-beam computed tomography. Am J Orthod Dentofacial Orthop. 2007;131(4 Suppl):S82-9.

4. Upadhyay M, Yadav S, Nanda R. Vertical-dimension control during en-masse retraction with mini-implant anchorage. Am J Orthod Dentofacial Orthop. 2010;138(1):96-108.

5. Wagner JD, Baack B, Brown GA, Kelly J. Rapid 3-dimensional prototyping for surgical repair of maxillofacial fractures: a technical note. J Oral Maxillofac Surg. 2004;62(7):898-901.

6. Huang J, Bumann A, Mah J. Three-dimensional radiographic analysis in orthodontics. J Clin Orthod. 2005;39(7):4218.

7. Hamada Y, Kondoh T, Noguchi K, lino M, Isono H, Ishii H, Mishima A, Kobayashi K, Seto K. Application of limited cone beam computed tomography to clinical assessment of alveolar bone grafting: a preliminary report. Cleft Palate Craniofac J. 2005;42(2):128-37.

8. Deguchi T, Nasu M, Murakami K, Yabuuchi T, Kamioka H, Takano-Yamamoto T. Quantitative evaluation of cortical bone thickness with computed tomographic scanning for orthodontic implants. Am J Orthod Dentofacial Orthop. 2006;129(6):721.e7-12.

9. Kim HJ, Yun HS, Park HD, Kim DH, Park YC. Soft-tissue and cortical-bone thickness at orthodontic implant sites. Am J Orthod Dentofacial Orthop. 2006;130(2):177-82. 
10. Poggio PM, Incorvati C, Velo S, Carano A. "Safe zones": a guide for miniscrew positioning in the maxillary and mandibular arch. Angle Orthod. 2006;76(2):191-7.

11. Schnelle MA, Beck FM, Jaynes RM, Huja SS. A radiographic evaluation of the availability of bone for placement of miniscrews. Angle Orthod. 2004;74(6):832-7.

12. Chun Y, Lim W. Bone density at interradicular sites: implications for orthodontic mini-implant placement. Orthod Craniofac Res. 2009;12(1):25-32.

13. Baumgaertel S, Hans MG. Buccal cortical bone thickness for mini-implant placement. Am J Orthod Dentofacial Orthop. 2009;136(2):230-5.

14. Lim J, Lee S, Kim Y, Lim W, Chun Y. Comparison of cortical bone thickness and root proximity at maxillary and mandibular interradicular sites for orthodontic mini-implant placement. Orthod Craniofac Res. 2009;12(4):299304.

15. Park J, Cho HJ. Three-dimensional evaluation of interradicular spaces and cortical bone thickness for the placement and initial stability of microimplants in adults. Am J Orthod Dentofacial Orthop. 2009;136(3):314.e1-12.

16. Melsen B, Bosch C. Different approaches to anchorage: a survey and an evaluation. Angle Orthod. 1997;67(1):2330.

17. Quinn RS, Yoshikawa DK. A reassessment of force magnitude in orthodontics. Am J Orthod. 1985;88(3):252-60.

18. Burstone CJ. The segmented arch approach to space closure. Am J Orthod. 1982;82(5):361-78.

19. Kuhlberg AJ, Burstone CJ. T-loop position and anchorage control. Am J Orthod Dentofacial Orthop. 1997;112(1):128.

20. Kanomi R. Mini-implant for orthodontic anchorage. J Clin Orthod. 1997;31(11):763-7. 